\title{
EL MODELO DE LA TRIPLE HÉLICE EN LA COMPRENSIÓN Y DESARROLLO DE PROYECTOS SOCIALES COMPLEJOS EN COSTA RICA
}

\author{
MARICRUZ CORRALES MORA \\ Universidad Estatal a Distancia, Costa Rica \\ moram@uned.ac.cr
}

\section{RESUMEN}

H. Etzkowitzy H. Leydesdorff, economista y sociólogo; respectivamente, recurren a una metáfora reconocida como la cadena helicoidal que representa al ADN en la ciencia, a la que han Ilamado "Triple Hélice", para representary entender la complejidad que se da de la interacción y colaboración necesarias entre tres sectores: gobierno, academia e industria. Con esto, se han promovido opciones de desarrollo económico, vinculadas a formas innovadoras de gestionar el conocimiento. En las siguientes páginas, se hace una sintesis de las razones y formas en que una Triple Hélice puede surgir en un país o región y por qué los autores sostienen que se crea una sinergia interinstitucional virtuosa, no solo en el campo de la innovación económica, sino también, en la comprensión y en el trabajo conjunto, que se da en la realización de proyectos sociales complejos que son de interés público.

PALABRAS CLAVE: INNOVACIÓN, TRIPLE HÉLICE, GOBIERNO, UNIVERSIDAD, INDUSTRIA.

\section{ABSTRACT}

Economist H. Etzkowitz and sociologist H. Leydesdorff used the helical DNA strands as a metaphor to represent and understand the complexity that arises from the necessary interaction and collaboration between three sectors: government, academia and industry. From a comprehensive combination of these sectors, the authors developed a model called the "Triple Helix" that promotes economic development based most often on innovative ways of knowledge management. A summary of the reasons and ways in which a Triple Helix can work in a country or region is presented, and the reasons why the authors argue that a virtuous synergy is created, not only in the field of economic innovation, but also in understanding and working a set of complex, social and public interest projects, are also analyzed.

KEYWORDS: INNOVATION, TRIPLE HELIX, GOVERNMENT, UNIVERSITY, INDUSTRY.

$$
\begin{aligned}
& \text { One needs metaphors to reduce } \\
& \text { the complexity for the discursive understanding } \\
& \text { (Necesitamos metáforas para reducir } \\
& \text { lo complejo de la comprensión discursiva) } \\
& \text { H. ETzKOWITZ Y H. LEYDESDORFF, (2000) }
\end{aligned}
$$

\section{INTRODUCCIÓN}

La llegada del siglo XXI supuso introducir a la cultura humana, en una nueva forma evolutiva sociocultural o era de desarrollo, que devino de la confluencia de diferentes corrientes o manifestaciones y, dieron lugar al período llamado Postmodernismo.

Conceptos y modelos de trabajo novedosos caracterizan a esta época, como lo son: "la sociedad del conocimiento"y"economía global"y, con ellos, se destacan al conocimiento (creación, aplicación y/o divulgación) como recurso y producto clave de la gestión socioeconómica; a las tecnologías de la comunicación e información, como moto- 
res de la innovación de procesos diversos y; a la competitividad, como estrategia productiva capaz de mejorar continuamente el mercado (Drucker, 2001; Castells, 2001; Dzisah, 2010).

En ese contexto sociocultural, las universidades están viviendo un proceso de transformación, bajo tres dimensiones diferentes: desde su naturaleza misma educativa, en la visualización de los desafíos que enfrenta y en su relativa impotencia para impactar de manera más efectiva a la sociedad (Jofré, 1998).

No obstante, el status único de la universidad, como institución que enseña, investiga y a la vez, contribuye con el desarrollo económico, la ubica en una posición central en esta era del conocimiento (Etzkowitz, 2011); especialmente, en relación con las demás instituciones productivas, de los sectores gobierno e industria.

Es por ello que, modelos conceptuales de análisis y trabajo multidimensionales, como la "Triple Hélice", pueden ayudar a reconocer la importancia económica del conocimiento, incrementar el uso de modelos sistémicos y aumentar el número de instituciones involucradas en la generación y uso de nuevos conocimientos (visión económica de la OECD; en Comisión Nacional para la Innovación, 2007).

Este artículo introduce dicho modelo, para tomarlo en cuenta en la realidad costarricense, a manera de instrumento de trabajo y, para la construcción de nuevos puentes intersectoriales, que han venido trabajando por su cuenta y que ahora, precisan integrarse, con el fin de obtener mejores resultados o respuestas a las demandas actuales.

\section{El modelo de la Triple Hélice}

En palabras de sus creadores, H. Etzkowitz (1993) con el trabajo conjunto de H. Leydesdorff (1995), el modelo de la Triple Hélice explica la necesidad de ampliar la vinculación industria y gobierno, propia de una sociedad industrial, a una interrelación tripartita creciente; con un nuevo actor: la universidad y, de ésta, con la industria y el gobierno, en la sociedad del conocimiento.

La tesis de esta visión es que el potencial para la innovación y el desarrollo económico, en una sociedad del conocimiento, reside en un rol más prominente que debe asumir la universidad, pero también, en la hibridación de elementos provenientes de ésta con la industria y el gobierno, de manera que, se permitan generar formas institucionales y estructuras sociales nuevas, para la producción, la transferencia y la aplicación del conocimiento (Ranga y Etzkowitz, 2013).

Es decir, se trata de una propuesta de modelo económico, basado en la capacidad compartida de estas tres organizaciones, para innovar su propio quehacer, el tipo y nivel de vinculación entre sí y procurar resultados, productos o procesos diferentes y mejores, ante los retos actuales complejos o, como lo señalan Etzkowitz y Viale (2010), para institucionalizar la innovación.

Esta concepción coincide con la idea de la dinámica natural de la innovación de Schumpeter ("la destrucción creativa" de la economía de 1942), al tener en cuenta que cuando el conocimiento es empleado progresivamente como un recurso para la producción y el sistema de distribución, todo acto de remodelación de la economía, que pueda llegar a prevalecer, lo hará como derivación de una "destrucción creativa" (Etzkowitz y Leydesdorff, 2000).

En ese contexto, es necesario aclarar que, tradicionalmente la universidad ha sido vista como una estructura de soporte para la innovación, al proveer profesionales en diferentes disciplinas o áreas y, ofrecer resultados de investigación y conocimiento a la industria, en general. Estas acciones las ha venido ejerciendo, de una u otra forma, durante décadas. Lo diferente que propone la Triple Hélice es que, además de formar 
e investigar (reproducción, resguardo, creación y divulgación de) conocimiento y cultura (el "ADN" del que se alimenta), la universidad desarrolle un nuevo formato académico empresarial (Etzkowitz, 2003; Etzkowitz y Viale, 2010). Uno capaz de unir/integrar el objetivo productivo de la industria y a las relaciones contractuales garantes dispuestas por el gobierno, para delimitar las interacciones e intercambios entre las partes, de forma que permitan mejorar las condiciones de innovación y la capitalización del conocimiento, en una sociedad basada en éste último (Viale y Ghiglione, 1998).

Se hace la salvedad de que lo expresado en el párrafo anterior, no supone eliminar o sujetar el principio de la autonomía universitaria (ampliamente defendido en el orbe), a los intereses industriales o del gobierno, sino que se trata de variar la visión institucional de universidad, para comercializar, en beneficio propio y de la sociedad, los procesos investigativos, los resultados, las aplicaciones y la posible transferencia tecnológica, además de estar más estrechamente involucrada con el desarrollo socioeconómico (Dzisah, 2010; Etzkowitz, 2003).

La idea básica de este esfuerzo tripartito, inter institucional y sectorial, no es del todo nueva.

Han existido otras posiciones al respecto (el Triángulo del Desarrollo Tecnológico de E. Sábato o el Nodo del Conocimiento de Cofone et al.), que intentaron explicar la vinculación potencial de la academia con los sectores productivos y el gobierno; pero ninguno aportó una visión sistémica de la sociedad y de los propios actores, para desarrollar una dinámica económica y organizacional. Por eso es que el concepto de interacción, sistematización, coordinación, complementación y ejecución de los procesos y acciones, desde una Triple Hélice de intervención conformada por dichos actores (similar a la representación visual dual del ADN; pero desde tres secuencias como sería la del ADN-H con en- laces de hidrógeno), rompe con el esquema tradicional de linealidad de los procesos y/o con las relaciones entre solo pares, para ofrecer una visión multidimensional, continua y dinámica, que no se detiene ni hace más importante a uno de los actores. Al contrario, los tres juegan su propio rol (gestor de conocimiento, productor y regulador geopolítico) y, entre sí, forman una organización compuesta (nuevo ser organizacional más complejo o hélice compuesta), para determinados fines o proyectos que, en sí, es inestable y, por consiguiente, requiere del esfuerzo ingente entre las partes, para poner en práctica procesos comunicativos, redes de trabajo, consensos y toma de decisiones, que permitan superar las diferencias insostenibles de intereses individuales y grupales de cada sector (Ranga y Etzkowitz, 2013).

Etzkowitz y Leydesdorff (2000) la definen en estos términos: la configuración de la Triple Hélice no presenta una sincronización a priori entre las hélices de los sectores que la conforman, ya que no se unen en un orden pre-establecido, sino más bien azaroso, según los intereses comunes que surgen y empiezan a trabajarse en forma conjunta.

La red de trabajo que se sostiene de esas relaciones primeras entre las hélices, genera una subdinámica reflexiva de intenciones, estrategias y proyectos, que añaden valor agregado a toda la composición, porque reorganizan y armonizan continuamente la infraestructura disponible entre todos, para alcanzar al menos una aproximación de las metas que se hayan establecido (Etzkowitz y Viale, 2010). Cabe destacar que este tipo de cadena tripartita se forma también cuando se enfrentan problemas en "clusters" industriales, o por vacíos propios de algún desarrollo académico o dada la pérdida de la autoridad de un gobierno.

La Figura 1 intenta representar la conformación por etapas del concepto antes descrito. 


\section{FIGURA 1 \\ REPRESENTACIÓN DE LA PROPUESTA MULTIDIMENSIONAL E INTERINSTITUCIONAL DE LA TRIPLE HÉLICE ENTRE UNIVERSIDAD, GOBIERNO E INDUSTRIA}

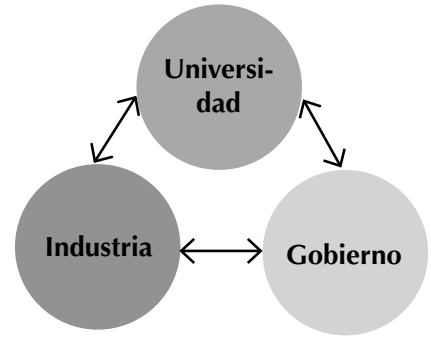

Actores independientes, con relaciones de colaboración recíprocas

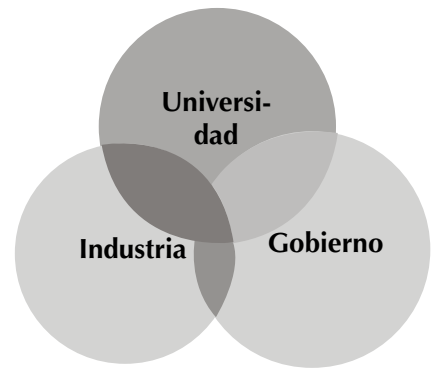

Colaboración con vínculos tripartitos iniciales

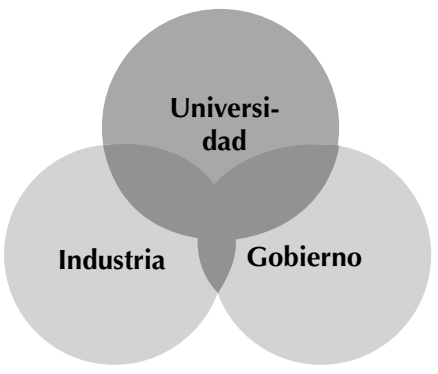

Fortalecimiento de la colaboración y surgimiento de la triple hélice

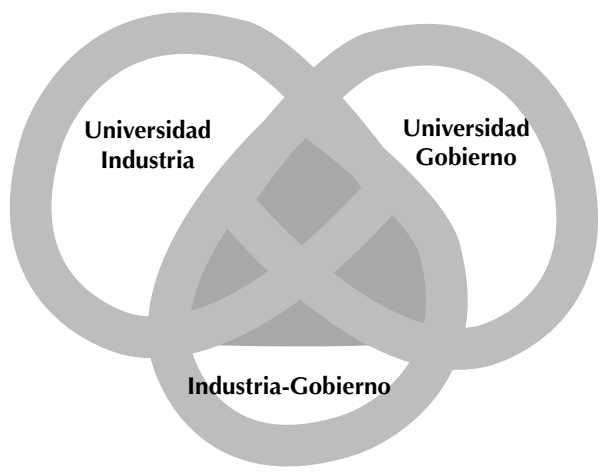

Representación transversal y específica de la triple hélice (vínculos duales y tripartitos

Fuente: Elaboración de la autora, a partir de representaciones de Etzkowitz y Leydesdorff (2000); Etzkowitz (2003) y Ranga y Etzkowitz (2013).

Esa conformación por etapas de la Triple Hélice también obedece a una interpretación desde la perspectiva institucional del concepto.

En el primer momento (esquina superior izquierda de la Figura 1), las representaciones de cada sector han partido desde una concepción representativa de modelo "laissez-faire", dado que ninguno de los sectores está inmerso en algún otro, como sí sucede cuando el gobierno asume el control total sobre las acciones del sector universitario e industrial (en un modelo estatista). Por eso, desde la figura superior izquierda, se representan las vinculaciones particulares que cada actor tiene con los demás y en las siguientes representaciones, se ofrece la descripción visual de la formación de la Triple Hélice, con la colaboración y el trabajo tripartito, según sea el campo de desarrollo e innovación (Etzkowitz y Leydesdorff, 2000; Ranga y Etzkowitz, 2013).

Al final con este tipo de experiencia interinstitucional compartida, lo que se busca es formular un ambiente innovador, formado por empresas universitarias emergentes, que comercian conocimiento; iniciativas trilaterales para el desa- 
rrollo económico basado en el conocimiento y; alianzas estratégicas entre firmas (grandes y pequeñas, operando en diferentes campos y con diferentes niveles de tecnología); laboratorios o instancias gubernamentales y grupos de investigación universitarios; todos ellos, haciendo frente a sub-dinámicas no lineales, propias de las fuerzas del mercado, poder político, controles institucionales, movimientos sociales, desarrollos tecnológicos y sistemas sociales, en general (Etzkowitz y Leydesdorff, 2000).

\section{Conformación de una Triple Hélice}

Desde el punto de vista de un mercado capitalista, esta clase de alianzas (entre gobierno, academia e industria) han surgido cada vez más, en el tanto la educación superior estatal ha visto mermar los recursos provenientes del gobierno para el logro de los objetivos y, ciencia e innovación (especialmente la tecnológica) se consideran motores para el desarrollo económico (Etzkowitz y Leydesdorff, 2000).

En especial, durante las últimas décadas, las universidades han sido presionadas por organismos internacionales (UNESCO, Banco Mundial; entre otros) y los gobiernos de turno, para que transformen los modelos institucionales de ingresos (dependientes de financiamiento estatal), en nuevas estrategias de venta de productos y servicios, provenientes de la actividad académica. Cuando la relación se da especialmente entre universidad e industria, se le ha conocido como "capitalismo académico" (término acuñado por S. Slaughter y L. Leslie en su libro homónimo publicado en 1997) y ha recibido fuertes críticas del propio sector académico, por romper con libertades centenarias de independencia de criterio y responsabilidad social por parte de las universidades (Mendoza, 2011).

El surgimiento de ese "capitalismo académico" tiene como punto de partida en Latinoamérica, la privatización de la educación superior en Chi- le, en la década de los ochenta que, por las circunstancias particulares de ese momento y sociedad, se tuvo que asumir en muy corto plazo, en detrimento de la vida universitaria y con una consecuente segmentación del sector educativo: algunas instituciones pudieron solo dedicarse a la docencia y muy pocas, a la investigación (Muñoz, 2013).

No obstante, a partir de esa experiencia, también surgieron otras consideraciones importantes de destacar, como la concentración de recursos en instituciones o departamentos con mejores rendimientos o servicios (especialmente en las áreas de ciencia y tecnología; en claro detrimento, muchas veces, de las áreas sociales) o la de una población estudiantil disminuida y molesta, por no tener más el estudio subvencionado de parte del estado. Lo primero, ha inducido a un cambio en la imagen y competitividad de las universidades, a nivel local e internacional, que se aprecia también, por el conocimiento del ejercicio académico, mediante las diferentes redes sociales y de telecomunicación existentes (Knight, 2002; World Reputation Rankings 2014). Lo segundo, se ha constituido en otra lucha social de las poblaciones con menores recursos, para acceder al conocimiento y a las oportunidades que brinda la educación en general.

En otro tipo de razonamiento, siempre en materia de financiamiento y contratación, se dice que la regla general es "quien paga, exige lo quiere". En la delimitación de los nuevos vínculos propuestos por este modelo de la Triple Hélice, ese principio no es del todo cierto, pues depende de la seriedad y respeto mostrado entre los actores involucrados (que ninguno busque la ventaja por sobre los demás), lo que cada uno aporta en la relación y en las metas esperadas con el vínculo, para que se cumpla o se deseche como principio básico (dando la libertad de seguir con la propia dinámica de cada quien, si es para bien de la interrelación) (Leydesdorff, 1996; Etzkowitz y Viale, 2010). 
Etzkowitz (2011) señala dos fases para que las universidades puedan iniciar la nueva gestión en venta de servicios o productos: la primera tiene que ver con el desarrollo interno de grupos de investigación consolidados que puedan comerciar o vender servicios a la sociedad (gobierno, industria y otros grupos), derivados de los resultados investigativos (diseño de "cuasi-firmas"). La otra fase tiene que ver con la participación académica en la internacionalización y capitalización del conocimiento, a través de productos tangibles y/o la venta de cursos de educación a distancia o virtuales.

En buena parte, el impulso a los investigadores para esta nueva tarea puede devenir del estímulo con premios y reconocimientos que les prodiguen más ingresos para continuar en su quehacer. De hecho, relaciones informales entre universidad e industria han existido a través del contacto entre profesores y los profesionales del área productiva que fueron alguna vez sus estudiantes. Sin embargo también ayuda que las licencias, contrataciones, servicios y cursos a distancia den valor al reconocimiento de las universidades, en su función natural de diseminar conocimiento.

Por otro lado, el reconocimiento de las ideas, como bienes económicos necesarios para generar crecimiento económico y desarrollo, genera un ciclo dinámico: entre más conocimiento, mayor capital y viceversa (Dzisah, 2010). Lograr esa nueva dinámica requiere de un cambio organizacional dentro de la universidad, no solo para superar los temores válidos que pueden surgir de los intereses económicos superiores a los objetivos de la academia (derechos de autor, patentes y otras formas de secreto comercial ejercidos sobre inventos/descubrimientos), sino porque deberán estar acompañados de nuevos protocolos, reglamentaciones y situaciones conductuales diferentes con los socios industriales y de gobierno (uno por el interés mercantil y el otro, por lo político) (Etzkowitz y Viale, 2010).
El éxito que logren y la continuidad de los vínculos en proyectos adicionales dependen del reconocimiento "del otro" (los otros sectores) y la importancia de la actividad que desarrollan en esa "participación compartida", mediante objetivos comunes y compromisos establecidos. Para ello, requieren de una re-interpretación y revisión de las reglas clásicas de funcionamiento escritas, que deberán elaborarse, con detalle, a partir de la experiencia conjunta que generen. Es decir, habrá que revisar situaciones como: qué puede legarse a la sociedad de manera gratuita, como avance del conocimiento y qué puede comercializarse; qué debe ser considerado de interés público y qué puede sumirse en el interés privado o, cuál será el mayor interés si la investigación básica o aplicada o ambas (Etzkowitz, 2011).

También, un liderazgo colaborativo se considera clave en toda asistencia emprendida e inclusive en la capacidad de moderación de conflictos, porque pueden conectar a las personas desde diferentes sectores, cimentar puentes, aportar visiones diferentes, generar consenso, balancear intereses particulares, hallar a los mejores profesionales para tareas o retos específicos, adelantarse a los cambios de pensamiento e implementaciones prácticas mediante la visión y reflexión y, crear nuevas oportunidades para el intercambio de conocimiento (Ranga y Etzkowitz, 2003).

\section{La Triple Hélice como sistema de innovación emergente}

En la consolidación sistémica de la Triple Hélice hay que tener presente los cambios que cada sector requiere y los obstáculos que deben superar como parte del involucramiento conjunto. A la vez, se esperan dos cambios importantes: que la universidad se convierta en una nueva institución creadora y propietaria de capital (intelectual más que físico). Por otro lado que el gobierno entre a escena como un ente em- 
prendedor; no solo para proveer de recursos a los otros dos actores (universidad e industria) o regular las relaciones de estas partes, sino que también pueda tener la capacidad de originar innovaciones organizacionales y ajustes estructurales, para posibilitar la innovación dentro del sistema económico (Dzisah, 2010; Etzkowitz y Viale, 2010).

Una primera visión sistémica de este modelo, planteado por Viale y Ghiglione (1998), concibe la representación final de estos tres mundos (lo académico, lo industrial y lo gubernamental) desde tres factores: los actores, las instituciones y las normativas (leyes o reglamentos de las partes). A cada factor, le asignaron un nivel de correspondencia: a los actores, el nivel "micro", porque son quienes se comportan de acuerdo con papeles y modelos de acción que implican culturas diversas, desde los cuales se inician los vínculos con los demás dominios. Las instituciones, en el nivel "meso", ya que utilizan sus organizaciones para organizarse con los demás ámbitos, en términos de posibles producciones y uso del conocimiento. Finalmente, un nivel "macro", en el que toma en cuenta a la normativa (leyes o reglamentos), ya que se considera esencial establecer directrices y definir incentivos a partir de regulaciones, que permitan la libertad a los actores e instituciones de establecer nexos de trabajo con los demás.

En otra concepción sistémica, Ranga y Etzkowitz (2013) señalan que la Triple Hélice puede verse como un conjunto de componentes, relaciones entre ellos y funciones.

Los componentes obviamente son las instancias institucionales universitarias, industriales y de gobierno que intervienen dentro de la acción tripartita. Algunos serán solo individuos, pero otros serán grupos, con la característica esencial de que se destacan como innovadores o que laboran en un área investigativa que puede ser de una o más disciplinas (unidimensional o multidimensional el campo de acción). Las relaciones se dan por transferencia tecnológica, colaboración, moderación de conflictos y trabajo en redes. Las funciones que conforman, pronto se transforman en las competencias que desarrollan como resultado de quienes participan, que por supuesto, en este contexto, tiene que ver con la generación, difusión y empleo de conocimiento e innovación; los espacios naturales de la concepción de una Triple Hélice.

Si para la concepción sistémica se asume una perspectiva evolutiva, desde la teoría social de sistemas de comunicación de Luhmann (las comunicaciones de individuos y grupos como unidad estructural de asociación e intereses compartidos), Ranga y Etzkowitz (2013) proponen concebir la Triple Hélice como un tipo de enlace entre universidad, industria y gobierno, que co-evoluciona en subconjuntos de sistemas sociales, con interacciones capaces de superponer redes recursivas de colaboración e, instancias que reformulan su propia institucionalidad, dinámicas y propio sistema, por formas de diferenciación nuevas en el trabajo, en las relaciones institucionales y en la selección de ambientes favorables (mercados o espacios de innovación), surgidos de los objetivos comunes que buscan alcanzar.

En otras palabras, según el campo disciplinario, los individuos y la experiencia acumulada en ellos y en cada institución (gobierno, universidad o industria), éstos se agrupan para investigar e innovar con objetivos comunes, rompiendo así los estándares usuales de vinculación interinstitucional entre ellos, que a la vez, termina influyendo en otros colegas o instancias para emularlos (recursividad), con lo que se dinamiza la relación y al sistema mismo.

Cuando estos sistemas progresan a un nivel de madurez y estabilidad suficientes, dentro de contextos culturales específicos, sean locales o regionales, tienden a concebirse solo como sis- 
temas de innovación y asumen otras configuraciones. Este es el caso de los parques científicos, de innovación o tecnológicos. A este nivel, las funciones se diferencian para dar lugar a cuatro tipo de capacidades: a) Estratégica o selectiva; b) Organizacional (para la integración y coordinación); c) Técnica o funcional y; d) Adaptativa a partir del aprendizaje acumulado (Ranga y Etzkowitz, 2013). Estas formas nuevas de presentación del funcionamiento de una Triple Hélice, siguen siendo realmente escasas, según los autores, por la complejidad y experiencia acumulada que requieren para su creación y consolidación.

Por otra parte, Viale y Pozzali (2010) también señalan la preocupación básica de todo gobierno, en materia de racionalizar las políticas públicas de desarrollo y cuando asumen un rol organizativo proactivo para el desarrollo y gestión del conocimiento: o lo hacen desde la formulación de políticas generales impuestas a los diferentes sectores o, definen y realizan gestiones (incentivos especialmente), para variar circunstancias y condiciones, que permiten a los sectores modificar su intervención / participación en los mercados.

La primera opción surge cuando se presentan "fallos del mercado", que pueden solo corregirse por la intervención del estado, dentro de una perspectiva de planeamiento directo y en la que el gobierno juega un rol preponderante, para reordenar las fuerzas y acciones del sistema social. Por el contrario, la segunda opción se favorece cuando la sociedad es compleja, crítica ante intervenciones externas y con suficientes medidas de control o transparencia de los procesos. En este último escenario, el desarrollo se da a través de redes, con actores del sistema social que juegan roles específicos en las relaciones que establecen y en las que se provocan cambios e incentivos, capaces de variar al propio sistema social. La conformación de la Triple Hélice con este tipo de ordenamiento social complejo se considera más natural o avanzado por los me- canismos de enlace y coordinación que puede aportar y la intervención especializada de cada actor, según las necesidades se los demanden. Viale y Pozzali (2010) lo terminan Ilamando sistemas adaptativos complejos (CAS por sus siglas en inglés, Complex Adaptative System).

La otra situación crítica por considerar son los conflictos de intereses de las partes involucradas para permitir la creación de una Triple Hélice, ya que en la universidad no surgen rápidamente los roles de estudiantes o profesores como nuevos empresarios; tampoco, la industria busca a la universidad más allá de los procesos de actualización y capacitación que demande para la producción. Ambas instituciones se requieren en situaciones complejas o claves por resolver. Sin embargo, es sólo de la universidad que se espera que llegue a relacionar el discurso académico con los problemas mundiales reales (Dzisah, 2011).

Por eso, es que de nuevo, la Triple Hélice puede generarse con más facilidad, en aquellos sistemas sociales cuyos actores han demostrado competitividad local e internacional. Esto se da gracias a tres factores específicos que deben tomarse en cuenta: las políticas locales de gobierno, el rol local de las universidades dentro del sistema de innovación regional y el grado de involucramiento de los actores privados. Es claro que en una visión centralizada, desde el funcionamiento institucional del gobierno, la universidad o la industria, no facilita la conformación de enlaces tripartitos (Viale y Pozzali, 2010). Se requiere al contrario formas de trabajo más descentralizadas y coordinadas.

También puede argumentarse que esa competitividad se desarrolla, en el tanto exista un cambio en el planteamiento educativo. Por ejemplo, los gobiernos pueden contribuir con el fortalecimiento de los planes y currículos en ciencias desde la escuela, para que desde temprana edad se estimulen las vocaciones científicas que lleguen a fortalecerse en las universidades. Sin 
este insumo previo, el componente académico seguirá siendo uno de los más débiles en la conformación de la Triple Hélice (Baber, 2001).

Conflictos adicionales en la Triple Hélice surgen por las tareas conducidas (funcionales, cognitivas o constructivas), que generan diferencias de opinión entre los departamentos funcionales de las organizaciones que están colaborando o, los conflictos relacionales (disfuncionales, afectivos o destructivos), que provienen de las personas y se generan por incompatibilidad entre diferentes personalidades, de diferentes departamentos, conduciendo a sentimientos negativos, tensiones y frustraciones. En este último aspecto, es claro que los conflictos interpersonales tienen un efecto negativo en el intercambio de conocimiento, al más alto nivel y en la toma de decisiones (Ranga y Etzkowitz, 2013), por lo que deberían evitarse por completo.

\section{CONCLUSIONES}

El modelo de la Triple Hélice todavía tiene muchos detractores en Costa Rica en lo particular y, en la región, en lo general; debido a varios factores no resueltos o poco discutidos. Por ejemplo, poco se ha avanzado en delimitar la composición y funcionamiento de sistemas; fuera de tomar ejemplos específicos y sistemas que han sido favorecidos por las circunstancias y condiciones (casos de lugares como Boston o Silicon Valley).

Además, el surgimiento de una Triple Hélice, depende de los intereses políticos y económicos predominantes en su conformación: No es lo mismo una vinculación basada en el interés público civil de la sociedad, que el caso del manejo político del interés por la supremacía bélica de un país, lo cual ha conducido a casos exitosos de Triple Hélice como en el MIT (Massachussetts Institute of Technology) o en la Universidad de Stanford; ni tampoco la reunión de condiciones e infraestructura presentes en sociedades que tienen un mayor desarrollo económico y tecno- lógico que otras (como se ha dado en la creación de los Parques científicos de Barcelona y de Singapur).

Desde la perspectiva del interés general productivo, Viale y Pozzali (2010) concluyen que es precisamente la diferencia en las herramientas cognitivas y de conocimiento, empleadas por los miembros del sistema social, lo que marca la diferencia entre diferentes propuestas del modelo de la Triple Hélice, ya que contar con ellas, es disponer de factores claves para reforzar procesos de innovación, adaptación y capacidad productiva. El ejemplo más conocido es el del Silicon Valley, con avanzada diferenciación en conocimiento, cultura, desarrollo de redes sociales, capacidad auto-organizativa (que a la vez fortalece creación de nuevos vínculos entre instancias universitarias, de gobierno y las industrias locales) e incentivos selectivos claros.

En otras palabras, es necesario que los creadores de políticas públicas estén conscientes de que no les corresponde directamente formular el plan de nacimiento de sectores industriales innovadores, con la colaboración del sector respectivo y la academia; sino más bien, les corresponde tener claridad en suplir lo necesario de infraestructura inicial, ambiente normativo y recursos humanos para contribuir con el acompañamiento de las demás partes. Es decir, del gobierno se espera un rol regulador, que permita superar las vicisitudes provenientes de las crisis endógenas y exógenas afrontadas por la academia e industria.

La otra limitación para conformar Triples Hélices tiene que ver con la idiosincrasia de cada sector, la cultura que han sostenido durante años y que cuesta modificarla para avanzar en formas de trabajo y colaboración interinstitucional nuevas, con fines compartidos. En el caso de la universidad, es asumir lo que se ha llamado en este ámbito económico una "tercera misión", pues se trata de la intervención más directa con 
el desarrollo económico local y regional, ya sea mediante procesos de comercialización del conocimiento o resultados de investigación aplicados o formulación de empresas ligadas a la academia (Etzkowitz y Leydesdorff, 2000; Etzkowitz y Viale, 2010).

Influye además, la normativa, procedimientos y procesos de reconocimiento a lo interno de cada universidad, que durante décadas siguen estando en función sólo de los asuntos y trámites internos y que, al verse en una nueva dinámica social e institucional, reaccionan con negativas, temores u obstáculos para variar en algo su quehacer. Por ejemplo, una barrera importante por superar, en este tipo de procesos interinstitucionales, es la legislación vigente, más allá de las voluntades individuales o grupales; sobre todo, los que interpretan resguardar principios derivados de la Constitución Política de la República de Costa Rica, que pueden limitar algunos procesos de apertura en el desarrollo de enlaces y nuevas formas de gestión del conocimiento (la consulta a la Sala Constitucional de la República de Costa Rica para algunos proyectos de ley, concesiones públicas o privadas y otras demandas).

En esta misma línea legal de revisión, podría considerarse la Ley n. ${ }^{\circ} 8422$, contra la corrupción y el enriquecimiento ilícito en la función pública de Costa Rica, la cual podría suponer la restricción de recursos públicos que se inviertan en actividades propias del sector privado, al establecer amplias condiciones de los alcances interpretativos del principio de probidad del funcionario público y los posibles conflictos de intereses, cuando se realiza alguna vinculación con el sector privado (Contraloría General de la República, 2007).

No obstante, en la misma ley se han hecho las salvedades y solo si prevalece el interés público dentro del proceso de vinculación y, la razonabilidad de las formas vinculantes de cada sector (academia, gobierno e industria), asumiendo cada parte la responsabilidad social y económi- ca que le corresponde y, acatando los deberes de imparcialidad, objetividad y transparencia en el ejercicio de sus cargos, tendientes a asegurar un correcto desempeño de los funcionarios públicos dentro del ejercicio de competencias, tareas y labores asignadas (es decir, cumpliendo con los deberes y obligaciones de carácter ético, aún en sus actividades privadas, de manera que no pueda ser desleal a la función pública que cumple), se alcanzaría otro nivel de madurez institucional, democrático y económico diferente.

También la Ley n. 6227 , General de la Administración Pública (1998), es otro instrumento legal en Costa Rica que interviene con acciones en la consolidación del modelo de la Triple Hélice, en materia de contratación y vínculos con el sector público costarricense, porque cubre a las universidades estatales al ser instituciones de Derecho Público.

En términos amplios, esta ley establece las responsabilidades de los actores en acciones administrativas (contrataciones de servicios o similares), que deben darse con la injerencia de funcionarios del más alto nivel (Ministro o Gerencias o Vicerrectorías) o con la incorporación de expertos en la materia (calificados oficialmente), de manera que en ningún caso se pierdan las potestades de imperio y su ejercicio (del Estado), los deberes públicos y su cumplimiento, porque éstos son calificados por la ley como irrenunciables, intransmisibles e imprescriptibles.

Otros temores igualmente surgen en el ámbito industrial, porque pueden asumir objetivos que están por encima del respeto entre profesiones o sectores; demandan resultados efectivos en menor tiempo y sostienen una dinámica organizacional que ejecuta procesos con rapidez, en relación a los demás componentes del modelo (gobierno o universidad).

Así mismo, cualquiera sea el componente, todos deben superar las fuentes potenciales de conflicto, tanto a nivel micro (en la interacción de los individuos) como a nivel meso (entre las 
instituciones, marcos legales, normas y regulaciones), básicamente con la aplicación de habilidades sociales como el diálogo, manejo de las expectativas, re-direccionamiento de los temores individuales, creación de espacios culturales compartidos (especialmente orientados al intercambio de conocimiento y mejores estrategias de monitoreo para los vínculos establecidos y la diseminación de las mejores prácticas de emprendimiento) y empoderamiento que puedan desplegar (Ranga y Etzkowitz, 2013).

Lo importante con una creación de una Triple Hélice, es la oportunidad que se abre para abrir/expandir espacios de conocimiento y resolución innovadora de problemas, mediante la co-participación de tres actores institucionales, que llegan a ser complementarios en una realidad compleja y multidimensional y que atrae a líderes, de cada uno de esos sectores, para conformar grupos de investigación e/o innovación, que antes estaban dispersos geográfica, organizativa y políticamente, alrededor de temas, cam- pos de acción científicos y sociales, resolución de problemas o crisis y formulación de planes compartidos.

Lo crítico para que se vean beneficiados con este modelo, un proceso tan complejo como la innovación y otro igualmente difícil como el de hallar estrategias efectivas para el desarrollo económico de una población, es que se transforme en una propuesta de trabajo colaborativo, institucional, dinámico y económicamente factible, en el que se pueda contribuir con la circulación de recursos y flujos de conocimiento, generación de espacios innovadores y de consenso, identificación de brechas y soluciones en áreas diversas, generación de espacios científicos articulados, avances en la teoría y práctica de la innovación (Ranga y Etzkowitz, 2013).

La Figura 2 resume las condiciones antes mencionadas para gestar la implementación de una Triple Hélice en alguna área innovativa, social o productiva.

\section{REPRESENTACIÓN SISTÉMICA DEL MODELO DE LA TRIPLE HÉLICE CON LOS NIVELES DE CORRESPONDENCIA ENTRE LOS ACTORES, INSTITUCIONES Y REGULACIONES}

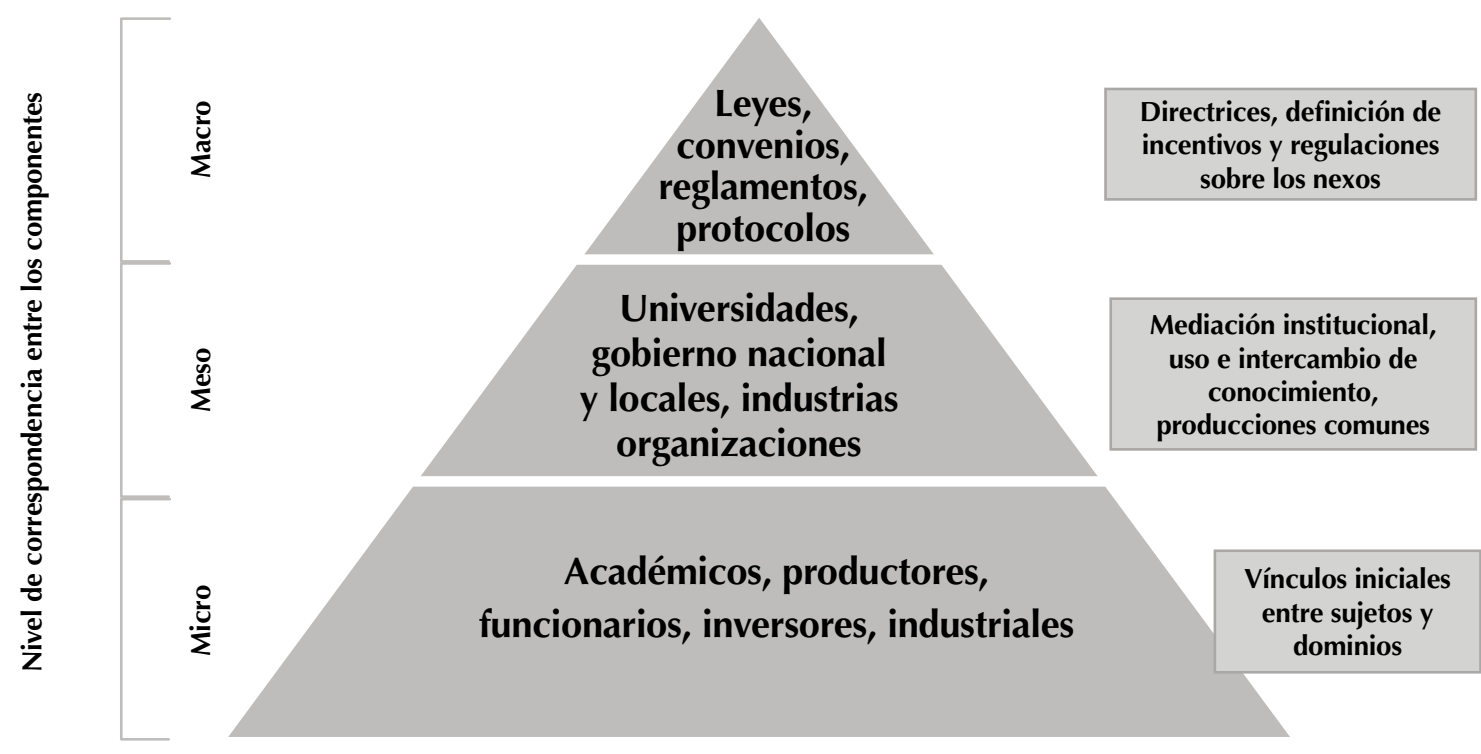

Fuente: Imagen elaborada por la autora a partir de lo descrito sobre la visión sistémica de Viale y Ghiglione (1998). 
En Costa Rica no se ha dado la voluntad (o decisión o condiciones) de las partes, para establecer una vinculación tripartita como la que se plantea en una Triple Hélice. Sin embargo, al menos existen experiencias exitosas bipartitas de gobierno-industria (leyes n. 8262 y 8634 , del Fortalecimiento de las Pequeñas y Medianas Empresas y Sistema de Banca para el Desarrollo), universidad-gobierno (asesorías de laboratorios universitarios en materiales y modelos estructurales para el control y calidad de construcción de obra pública LANAME-UCR y Ministerio de Obras Públicas y Transporte) y, universidadindustria (asesorías en la tecnología de alimentos industriales del CITA-UCR a industrias); que siguen siendo experiencias no generalizadas en las áreas del conocimiento o en todo un centro educativo de educación superior público o privado, pero que han dado muestra de efectividad en el mediano plazo.

De hecho, esos casos responden a un primer nivel de vinculación que sería solo disponer de una doble hélice.

La tercera hélice requiere de una especialización en los campos disciplinarios y en la gestión interinstitucional, que permite la vinculación tripartita: al inicio en modo experimental o piloto, y luego, superando las muchas barreras antes mencionadas (normativas, institucionales, actitudinales o de gestión del conocimiento), hasta llegar a lo que plantea este modelo de la Triple Hélice, con opciones viables y sostenibles, que se destacan en la conformación de parques tecnológicos o científicos.

En este punto, un primer ejercicio se dio en la región centroamericana y del Caribe, cuando en la pasada década, empresarios, autoridades del gobierno y de la universidad, recibieron una capacitación regional y nacional para la formulación y creación de Parques Tecnológicos y Científicos (iniciativa generada por el Ministerio de Ciencia y Tecnología), con la invitación de exper- tos internacionales y con el apoyo de programas de las Naciones Unidas y del gobierno chino (Murillo, 2008). Como iniciativa fue importante la capacitación recibida y los esfuerzos de colaboración internacional, pero en lo que respecta a la academia costarricense, se necesita mayor liderazgo conjunto entre universidades y bastante de lo diseñado, se ha archivado (Murillo, agosto 2010).

En Costa Rica, los esfuerzos para este tipo de alianzas corren todavía por cuenta de iniciativas separadas, bastante individualizadas, porque más bien predomina el ángulo de la ventaja competitiva de la individualidad (la ventaja de ganancia para solo una de las partes), en lugar de darse el aporte serio y responsable desde la institucionalidad (sea por parte del gobierno, las universidades estatales o del sector privado). De esta manera, proyectos de infraestructura vial, comunales, regionales o proyectos de importancia para el país, se han visto detenidos, desfasados, intervenidos y reformulados a destiempo, dando al traste con muchos esfuerzos no integrados, poblaciones e ilusiones que pudieron hacerse realidad, si se hubieran superado las limitaciones.

El país ciertamente requiere de infraestructura física y política pública renovada, pero sobre todo, de un cambio de mentalidad en las formas posibles de trabajo en conjunto, en el reconocimiento de los potenciales de las otras partes (sectores, organizaciones, instituciones) y en la credibilidad de sostener programas y acciones a partir de la injerencia, experiencia y aportes de conocimiento tripartitos de la academia, la industria y el gobierno.

Por tanto, la posible formulación de una Triple Hélice en Costa Rica parece estar todavía lejos de implementarse, sin antes superar algunas de las anteriores limitaciones previsibles entre la academia, el gobierno y el sector productivo, desde el actual planteamiento interinstitucional costarricense. 
Lo anterior, también demora toda aplicación de este modelo y de la sinergia interinstitucional virtuosa que podría generar, más allá del campo de la innovación económica de donde surgió, para que pueda ser aplicado igualmente en la realización de proyectos sociales complejos, que sean de interés público.

En términos generales, hace falta gestar un siguiente nivel de integración, que en el contexto costarricense puede significar más sostenibilidad inclusive para proyectos complejos, en donde no basta la intervención del Estado, ni la colaboración universitaria, si no se puede incluir también al sector productivo local o regional, para hacer mayor la influencia, cobertura y sostenibilidad del proyecto, en cuestión.

Esto es precisamente lo que no se dio en la formulación de la Ley n. ${ }^{\circ} 8725$, conocida como Proyecto Limón Ciudad Puerto (1999), en la que por ejemplo, no se definió participación alguna del sector privado o industrial, la implementación total del proyecto fue definida por y para instancias gubernamentales (14 en total, sin coordinación previa alguna y mucho menos formas colaborativas anteriores de trabajo con un fin en común) y la convocatoria para las universidades fue prácticamente inexistente (Contraloría General de la República, Febrero 2012). En ese contexto, el objetivo general de potencializar el desarrollo de la ciudad de Limón, mediante la creación de sinergias entre la ciudad revitalizada y el puerto marítimo moderno y eficiente, con generación de servicios, empleo e inversiones que dé lugar al mejoramiento de la calidad de vida de los habitantes, se torna inviable.

¿Este tipo de proyectos sociales complejos y de interés público podrían resolverse con la Triple Hélice?

Si se definen los proyectos sociales complejos y de interés público resultantes de procesos sistémicos que presenten: a) Énfasis interdisciplinario y/o transdisciplinario para realizar cambios significativos o innovaciones en las relaciones sociales de una comunidad, región o sociedad; b) Implementación de procesos de movilización-participación; c) Resultados de acciones que alcancen mejoras en las relaciones sociales, las estructuras de gobierno; d) Mayor empoderamiento colectivo y otras manifestaciones colectivas sociales (Moulaert, MacCallum y Hillier, 2013), se está ante la necesidad de una estructuración y sistematización como la que se ha descrito que conlleva la Triple Hélice.

Además, el status especial de la universidad, desde la dinámica de las sociedades basadas en el conocimiento, como institución que enseña, investiga y a la vez, tiene el mandato de contribuir con el desarrollo económico básico de una nación, le confiere una importancia crucial, para saber cómo intervenir en la formulación y en el desarrollo de procesos conducentes a la mejora de las condiciones de vida de diferentes poblaciones (al estilo de una productora y promotora de ideas, que al ser el nuevo bien económico necesario, la inclina a ser una institución líder del ciclo dinámico expresado por "a mayor conocimiento, mayor capital") (Dzisah, 2010).

Este tipo de dedicación hacia lo social es lo que podría llamarse el refuerzo a las funciones de servicio a la sociedad, a la cooperación con el mundo del trabajo y al análisis y previsión de las necesidades de la sociedad; mandato establecido en la Declaración Mundial sobre la Educación Superior en el siglo XXI (UNESCO, 1998), que se convierte en la razón por la que la universidad podría empezar a liderar también proyectos sociales de interés público.

Sin embargo, a pesar de ello, la intervención de las universidades en proyectos de servicio público en general, se ha visto actualmente reducida, debido a fuerzas de carácter político-financiero (UNESCO, 1998; entre otros), que se expresan en las presiones de los gobiernos de turno, para que redoblen esfuerzos en desarrollar nuevas 
estrategias con los que puedan cubrir los costos crecientes de su gestión. Así, con la venta de productos y servicios, provenientes de la actividad académica, obtiene réditos importantes que le permiten sostener la injerencia universitaria más allá de su misión natural de formar e investigar, según le dicte la aplicación del derecho de autonomía que le asiste (Altbach, Reisberg y Rumbley; 2009).

El punto es que tampoco las universidades, por sí solas, deben ser las únicas instituciones responsables en atender proyectos de índole social, sino también, que por supuesto el gobierno y algunos de los sectores productivos afines a la problemática social específica.

Ninguna de las partes, sea la academia, el gobierno o la industria, puede atender este tipo de proyectos sociales por sí misma, sino recurre a la interdependencia de funciones entre ellos, con lo que podrían alcanzar mejores y mayores logros.

La complejidad sorprende y supera toda injerencia individualizadora de un único actor social productivo, como parece estar demostrando la práctica de intervenciones o logro hasta un nivel de doble hélice, con el que han tenido éxito relativo las organizaciones en asociaciones bipartitas.

De ahí que, un modelo estructurante como la Triple Hélice, Ilegue a ser considerado un recurso conceptual y metodológico, con el potencial de conducir a posibles soluciones, en la implementación de proyectos sociales rentables o sostenibles.

Lo crucial es superar el margen de distancia que todavía yace en las mentes de los actores, las historias institucionales y los vacíos legales, para disponer de innovaciones organizacionales, arreglos y redes entre instituciones, adecuadas formas de transferencia tecnológica o conocimiento en general y, reorganización de sectores sociales y pro- ductivos, capaces de transformar y/o generar una nueva calidad de vida sostenible.

\section{REFERENCIAS}

Altbach, P. G., Reisberg, L. y Rumbley, L. E. (2009). Tras la pista de una revolución académica: Informe sobre las tendencias actuales. Resumen. Conferencia Mundial sobre la Educación Superior, organizada por la UNESCO, Francia. Recuperado en

http://unesdoc.unesco.org/images/0018/001831/183168s. pdf

Asamblea Legislativa (15 de octubre de 1998). Ley General de la Administración Pública. San José, Costa Rica. Recuperada en http://documentos.cgr.go.cr/content/ dav/jaguar/documentos/normativa/ley_gen_admon_pub/ley_gral_admon_publica.htm

Asamblea Legislativa. (10 de junio de 2009). Aprobación del Contrato de Préstamo n. ${ }^{\circ} 7498-C R$ y sus Anexos, entre el Gobierno de la República de Costa Rica y el Banco Internacional de Reconstrucción y Fomento (BIRF) para financiar el Proyecto de Limón Ciudad-Puerto. San José, Costa Rica. Recuperada en http://www.limonciudadpuerto. go.cr/index.php/2013-08-12-22-55-19/ley

Baber, Z. (Octubre, 2001). Globalization and scientific research: the emerging Triple Helix of state-industryuniversity relations in Japan and Singapore. Bulletin of Science Technology \& Society, 21 (401). DOI: 10.1177/027046760102100509.

Castells, M. (2001). The Internet galaxy. Reflections on the Internet, business and society. Devon, Great Britain: MPG Books Group, Bodmin and King's Lynn.

Centro Interuniversitario de Desarrollo (Junio del 2007). Educación superior en Iberoamérica. Informe 2007, 1a. Ed. Santiago, Chile: CINDA.

Comisión Nacional para la Innovación (2007). Atlas para la innovación en Costa Rica. Informe final. San José, Costa Rica: MICITT.

Contraloría General de la República y Procuraduría General de la República (Noviembre 2007). Ley contra la corrupción y el enriquecimiento ilícito en la función pública. Anotada y concordada. San José, Costa Rica: Publicaciones de la Contraloría General de la República. Recuperada en http://documentos.cgr.go.cr/content/ dav/jaguar/documentos/cgr/asesoria/LeyContralacorrupcion/Ley\%20Contra\%20la\%20Corrupci\%c3\%b3nweb.pdf 
Contraloría General de la República y Procuraduría General de la República . (20 de febrero, 2012). Informe del estudio sobre la ejecución del proyecto Limón ciudad-puerto. Informe n. DFOE-SAF-IF-03.2012. División de Fiscalización Operativa y Evaluativa. Área de Fiscalización del Sistema de Administración Financiera de la República. San José, Costa Rica.

Drucker, P. F. (Mayo, 1983). Modern prophets: Schumpeter and Keynes? Forbes. Recuperado de: http://druckersociety.at/files/p_drucker_proph_en.pdf

Drucker, P.F. (Noviembre 1, 2001). The next society. En The Economist. Recuperado en http://www.economist. com/node/770819

Dzisah, J. (2010). Capitalizing knowledge: the mind-set of academic scientists. Critical Sociology 36(4) 555-573. DOI: $10.1177 / 0896920510365917$.

Etzkowitz, H. (2003). Innovation in innovation: the Triple Helix of university-industry-government relations. Social Science Information, 42(293). DOI: 10.1177/05390184030423002.

Etzkowitz, H. (2011). Normative change in science and the birth of the Triple Helix. Social Science Information, 50(3-4) 549-568. DOI: 10.1177/0539018411411403.

Etzkowitz, H. y Leydesdorff, L. (2000). The dynamics of innovation: from National Systems and "Mode 2" to a triple helix of university - industry - government relations. Research Policy 29, p. 109-123. Recuperado de http:// cmapspublic3.inmc.us/rid=1223538615937_141997 1854_1861/etzkowitz-innovation\%20triple\%20helix. pdf

Etzkowitz, H. y Viale, R. (2010). Polyvalent knowledge and the entrepeneurial university: a third academic revolution? Critical Sociology, 36(4), pp. 595-609. DOI: 10.1177/0896920510365921.

Faure, E., Herrera, F., Kaddoura, A., Lopes, H., Petrovski, A. V., Rahnema, M., Ward, F. C. (1972). Aprender a ser. La educación del futuro. (V. española de C. Paredes). Recuperado de http://unesdoc.unesco.org/ images/0013/001329/132984so.pdf

Fontan, J. M., Harrinson, D. y Klein, J. L. (2013). Partneshipbased research: coproduction of knowledge and contribution to social innovation. The International Handbook on Social Innovation, , pp. 308-319, Cheshire, UK: MPG PrintGroup, UK.
Jofré, A. (1998). La universidad en América Latina. Desafíos y estrategias para las próximas décadas. 2a. Ed. Cartago, Costa Rica: Editorial Tecnológica de Costa Rica.

Knight, J. (2002). Trade in higher education services: The implications of GATS. Report. The Observatory on Borderless Higher Education. United Kingdom. Recuperado de http://www.unesco.org/education/studyingabroad/ highlights/global_forum/gats_he/jk_trade_he_gats_ implications.pdf

Leydesdorff, L. (1996). Luhmann's sociological theory: its operationalization and future perspectives. Social Science Information (35):283. DOI: 10.1177/053901896035002007. Recuperado de http://ssi.sagepub.com/content/35/2/283

Mendoza, P. (2011). El capitalismo académico en el cuadrante de Pasteur. Recuperado de http://education.ufl.edu/ mendoza/files/2011/02/JFHE-v33n3.pdf

Moulaert, F., MacCallum, D. y Hillier, J. (2013). Social innovation: intuition, precept, concept, theory and practice. En Moulaert, F.; MacCallum, D.; Mehmood, A. y Hambouch, A. (2013) (Eds), The International Handbook on Social Innovation. Collective Action, Social Learning and Transdisciplinary Research, pp. 13-24, United Kingdom: Edward Elgar Publishing Lmtd.

Muñoz, L. (30 de setiembre, 2013). Políticas públicas de financiamiento de la educación superior: implicaciones para la universidad pública. Revista Actualidades Investigativas en Educación, 13(3). Recuperado en http:// revista.inie.ucr.ac.cr/uploads/tx_magazine/politicaspublicas-financiamiento-educacion-superior-implicaciones-universidad-publica-munoz.pdf

Murillo, E. (29 de abril del 2008). Misión china y MICIT evaluaron condiciones para instalar parque tecnológico. Club de Investigación Tecnológica. Noticias. Recuperado en http://www.clubdeinvestigacion.com/contenido/ articles/misi\%C3\%B3n-china-y-micit-evaluaron-condiciones-para-instalar-parque-tecnol\%C3\%B3gico. html

Murillo, M. (Agosto, 2010). Parques tecnológicos estancados. Mayoría de proyectos en papel. El Financiero, edición 781. Sección Tecnología.

Postmodernism (2014). In Encyclopedia Britannica. Recuperado de http://www.britannica.com/EBchecked/ topic/1077292/postmodernism

Ranga, M. y Etzkowitz, H. (Agosto, 2013). Triple helix systems: an analytical framework for innovation policy and practice in the knowledge society. Industry \& 
RNA Revista Nacional de Administración

Higher Education, 27(3) pp. 237-262. DOI: 10.5367/ ihe.2013.0165

Tünnermann, C. (2003). La universidad ante los retos del siglo XXI. Mérida. México: Universidad Autónoma de Yucatán.

UNESCO (9 de octubre del 1998). Declaración mundial sobre la educación superior en el siglo XXI: Visión y acción. En Conferencia Mundial sobre la Educación Superior. Recuperada de http://www.unesco.org/education/educprog/wche/declaration_spa.htm

Viale, R. y Ghiglione, B. (1998). El modelo de triple hélice: una herramienta para el estudio de los sistemas socioeconómicos regionales europeos. En IPTS Report, 29. Recuperado en http://libros-revistas-derecho.vlex.es/vid/ triple-helice-socioeconomicos-regionales-117510

Viale, R. y Pozzali, A. (Julio, 2010). Complex adaptive systems and the evolutionary triple hélix. En Critical Sociology 36(4) pp. 575-594. DOl: 10.1177/0896920510365923.

World Reputation Rankings. En Times Higher Education (THE). London, UK. Recuperado de http://www. timeshighereducation.co.uk/world-university-rankings/2013-14/world-ranking

Recibido: 25 de marzo de 2014

Aceptado: 20 de abril de 2014 